\title{
Comparative transcriptome analysis during seeds development between two soybean cultivars
}

\author{
Li Peng ${ }^{\text {Equal first author, } 1}$, Linlin Qian ${ }^{\text {Equal first author, }{ }^{1} \text {, Meinan Wang }}{ }^{1}$, Wei Liu ${ }^{1}$, Xiangting Song ${ }^{1}$, Hao Cheng ${ }^{1}$, Fengjie Yuan \\ 2, Man Zhao ${ }^{\text {Corresp. } 1}$ \\ 1 college of bioengineering and biotechnology, zhejiang university of technology, hang zhou, China \\ 2 Institute of Crop Science, Zhejiang Academy of Agricultural Sciences, hang zhou, China \\ Corresponding Author: Man Zhao \\ Email address: mzhao@zjut.edu.cn
}

Soybean is one of the important economic crops, which supplies a great deal of vegetable oil and proteins for human. The content of nutrients in different soybean seeds is different, which is related to the expression of multiple genes, but the mechanisms are complicated and still largely uncertain. In this study, to reveal the possible causes of the nutrients difference in soybeans $A 7$ (containing low oil and high protein) and A35 (containing high oil and low protein), RNA-seq technology was performed to compare and identify the potential differential expressed genes (DEGs) at different seed developmental stages. The results showed that DEGs mainly presented at the early stages of seeds development and more DEGs were up-regulated at the early stage than the late stages. Gene Ontology and Kyoto Encyclopedia of Genes and Genomes analysis showed that the DEGs have diverged in A7 and A35. In A7, the DEGs were mainly involved in cell cycle and stresses, while in A35 were the fatty acids and sugar metabolism. Specifically, when the DEGs contributing to oil and protein metabolic pathways were analyzed, the differences between A7 and A35 mainly presented in fatty acids metabolism and seeds storage proteins (SSPs) synthesis. Furthermore, the enzymes, fatty acid dehydrogenase 2 (FAD2), 3-ketoacyl-CoA synthase (KCS) and 9S-lipoxygenase (LOX), in the synthesis and elongation pathways of fatty acids, were revealed probably to be involved in the oil content difference between A7 and A35, the SSPs content might be due to the transcription factors: Leafy Cotyledon 2 (LEC2), and Abscisic acid-intensitive 3 (ABI3), while the sugar transporter, SWEET10a, might contribute to both oil and protein content differences. Finally, six DEGs were selected to analyze their expression using qRT-PCR, and the results were consistent with the RNA-seq results. Generally, the study provided a comprehensive and dynamic expression trends for the seed development processes, and uncovered the potential DEGs for the differences of oil in A7 and A35. 


\section{Comparative Transcriptome Analysis during Seed Development in Two Soybean Cultivars}

3 Li Peng ${ }^{1 \#}$, Linlin Qian ${ }^{1 \#}$, Meinan Wang ${ }^{1}$, Wei Liu ${ }^{1}$, Xiangting Song ${ }^{1}$, Hao Cheng ${ }^{1}$, Fengjie 4 Yuan $^{2}$, Man Zhao ${ }^{1 *}$

51 College of Bioengineering and Biotechnology, Zhejiang University of Technology, 310014

6 Hangzhou, China;

72 Institute of Crop Science, Zhejiang Academy of Agricultural Sciences, 310014 Hangzhou, 8 China

9 \#co-first authors: these authors contributed equally

*Correspondence: mzhao@zjut.edu.cn; Tel./Fax:+86-0571-88320781

12 Author email list:

13 L.P.: 15182613887@163.com

14 Q.L.: 1677325557@qq.com

15 M.W.: 2317532770@qq.com

16 W.L.: 1197028959@qq.com

17 X.S.: 292365976@qq.com

18 H.C.: 1124733507@qq.com

19 F. Y.: fjyuanhz@126.com

20 M.Z.: mzhao@zjut.edu.cn

21 Running title: Transcriptome research in soybean

22

ABSTRACT: Soybean is one of the important economic crops, which supplies a great deal of 24 vegetable oil and proteins for humans. The contents of nutrients in different soybean seeds are 25 different, which is related to the expression of multiple genes, but the mechanisms are complicated and still largely uncertain. In this study, to reveal the possible causes of the nutrient

27 differences in soybean cultivars A7 (containing low oil and high protein) and A35 (containing 28 high oil and low protein), RNA-seq technology was performed to compare and identify the 
29 differentially expressed genes (DEGs) at different seed developmental stages. The results 30 showed that differential gene expression mainly occurred during the early stages of seed 31 development and more DEGs were up-regulated during the early stages than during late stages. 32 Gene Ontology and Kyoto Encyclopedia of Genes and Genomes analysis showed that the DEGs 33 have diverged in A7 and A35. In A7, the DEGs were mainly involved in cell cycle and stresses, 34 while in A35 they were involved in the fatty acid and sugar metabolism. Specifically, when the 35 DEGs contributing to oil and protein metabolic pathways were analyzed, differences between A7 36 and A35 were mainly observed in fatty acid metabolism and seed storage protein (SSP) synthesis. 37 Furthermore, the enzymes fatty acid dehydrogenase 2 (FAD2), 3-ketoacyl-CoA synthase (KCS) 38 and 9S-lipoxygenase (LOX), involved in the synthesis and elongation pathways of fatty acids, 39 were found to be related to the oil content difference between A7 and A35. The SSP content 40 might be affected by the transcription factors Leafy Cotyledon 2 (LEC2) and Abscisic acidintensitive 3 (ABI3), while the sugar transporter, SWEET10a, might contribute to both oil and protein content differences. Finally, six DEGs were selected to analyze their expression using qRT-PCR, and the results were consistent with the RNA-seq results. Generally, the study provided a comprehensive overview of the dynamic expression trends during seed development, and uncovered the potential DEGs responsible for the differences in oil content in A7 and A35.

Subjects Plant biology, Comparative transcriptome

Keywords soybean; comparative transcriptome; differential gene expression; oil; proteins

\section{INTRODUCTION}

Soybean (Glycine max L. Merr) is a staple economic crop, which provides about one-third of the protein and vegetable oil for human diet (Graham and Vance, 2003). The contents of oil and protein vary among soybean varieties, with seed oil and protein ranges from $15-25 \%$ and $35-50 \%$, respectively (Hurburgh, 1994). Soybean oil is mainly composed of unsaturated oleic, linoleic and linolenic acid, and their proportion determines the quality of the oil. SSPs in soybean, the other important nutrient, are composed of $\beta$-conglycinin (7S) and glycinin (11S) (Harada et al., 1989; Nielsen et al., 1989; Meinke et al., 1981). In soybean breeding, one of the important objects is to improve the contents of oil and protein. However, owing to the significantly negative correlation 
58 between oil and protein contents, it is difficult to develop soybean lines with high content of both 59 nutrients (Hyten et al., 2004a,b; Li et al., 2018a,b; Wilcox and Shibles, 2001).

60 In plants, seed development is a dynamic process, which is regulated by both genetic and 61 environmental factors. The accumulation of the nutrients oil, carbohydrate and protein, is 62 regulated by the programmed expression of a metabolic network during seed development 63 (Gupta et al., 2017; Le et al., 2007). Numerous studies have been performed to reveal the genetic 64 basis of seed development (Akond et al., 2014; Lee et al., 2007; Hwang et al., 2014; Hills, 2004). 65 For example, the studies of quantitative trait loci (QTL) and genome-wide association studies 66 (GWAS) have identified more than 100 QTLs related to the oil and protein contents, which are 67 widely distributed over the 20 soybean chromosomes (Hyten et al., 2004a,b; Akond et al., 2014; 68 Lee et al., 2007; Korte and Farlow, 2013; Panthee et al., 2006; Phansak et al., 2016; Wang et al., 69 2014). Furthermore, the sugar transporter GmSWEET10a which conferred simultaneous 70 increases in soybean seed size and oil content as well as reduction in protein content, was 71 identified through resequencing 800 genotypes (Wang et al., 2020).

72 The final chemical seed composition is a consequence of gene expression during seed 73 development. Along with the development in "omics"-areas, the genes and metabolites that are 74 required for seed development have been studied systematically (Li et al., 2015). Analyses of 75 gene expression during seed development have provided clues for understanding final seed 76 composition in different species, for example, Arabidopsis thaliana (Ruuska et al., 2002; 77 Palovaara et al., 2013; Fait et al., 2006), Medicago truncatula (Fedorova et al., 2002; Gallardo et 78 al., 2007), Brassica napus (Li et al., 2005), rice (Lan et al., 2005; Furutani et al., 2006), barley 79 (Watson et al., 2005), and soybean (Collakova et al., 2013). In soybeans, transcriptome analysis 80 of different developmental stages of seeds revealed that the most abundantly expressed genes 81 contributing to the metabolism and accumulation of oil and SSPs mainly occurred at the middle 82 and late stages (Li et al., 2015; Libault et al., 2010; Severin et al., 2010). For example, during the 83 synthesis of SSPs, the 7S and 11S SSPs were highly expressed at the late stage, a process 84 regulated by many transcription factors (Severin et al., 2010; Verdier et al., 2008). On the other 85 hand, many synthesis genes of amino acids and genes encoding proline-rich proteins were highly 86 expressed at the early seed development stages. As for lipids, there was a programmed 87 expression of lipid biosynthesis-related genes, in which FAD2-2B and FAD2-2C were highly 88 expressed at early stages and FAD2-1A and FAD2-1B were highly expressed at later stages 
89 (O’Rourke et al., 2014). GWAS analysis revealed that Glyma.11G229600.1 was positively and 90 Glyma.04G102900.1 was negatively correlated with the oleic acid content in soybeans, (Liu et 91 al., 2020).

92 In this study, RNA-Seq technology was applied to study three stages of seed development in two 93 different soybean cultivars with distinct protein and oil content. Through comparative 94 transcriptome analysis of developing seeds, we tried to reveal dynamic gene expression trends, 95 and identify the important DEGs and metabolic pathways involved in the accumulation of 96 nutrients. Finally, this study reveals the possible divergence of oil and protein accumulation 97 mechanisms in soybeans.

\section{MATERIALS AND METHODS}

\section{Plant materials}

101 Soybean plants of the cultivars A7 (Yudou 12) and A35 (Fendou 53) were grown and total RNA 102 was extracted from seeds as described in our previous research (Zhao et al., 2018). For each stage, three independent RNA extractions were performed and used in RNA-seq and qRT-PCR experiments.

\section{Determination of protein and oil contents of seeds}

106 Determination of the oil content in soybean: the mature seeds were ground to powder which was 107 transferred into $10 \mathrm{~mL}$ glass tubes. Oil was extracted with ligarine and total lipids (TL) were determined (Dong et al., 2001). The oil content of the soybeans was calculated on the basis of dry weight of the seeds. The content of proteins was determined by Kjeldahl's method. One gram of fine seed powder was used for digestion, cooling and distillation-titration. The percentage of bean protein was the total nitrogen percentage multiplied by 6.25. Each experiment was performed three times.

\section{cDNA library construction and transcriptome sequencing}

114 The total RNA (2 $\mu \mathrm{g}$ ) was sent to GENEWIZ (SuZhou, China) for sequencing, assembly and 115 clustering analysis. The total RNA was quantified and qualified using an Agilent 2100

116 Bioanalyzer (Agilent Technologies, Palo Alto, CA, USA) and a NanoDrop system (Thermo 117 Fisher Scientific Inc.). One $\mu$ g total RNA with RIN value above 7 was used for cDNA library 
118 preparation. First, double-strand cDNA was synthesized, and then treated to repair both ends and

119 add a dA-tailing, followed by a T-A ligation to add adaptors to both ends. Size selection of

120 adaptor-ligated DNA was then performed using AxyPrep Mag PCR Clean-up (Axygen), and

121 fragments of $\sim 360 \mathrm{bp}$ (with the approximate insert size of $300 \mathrm{bp}$ ) were recovered. Each sample

122 was then amplified by PCR for 11 cycles using P5 and P7 primers, with both primers carrying

123 sequences which can anneal to the flow cell to perform bridge PCR, and with the P7 primer

124 carrying a six-base index allowing for multiplexing. The PCR products were cleaned up using

125 AxyPrep Mag PCR Clean-up (Axygen), validated using an Agilent 2100 Bioanalyzer (Agilent

126 Technologies, Palo Alto, CA, USA), and quantified by Qubit 2.0 Fluorometer (Invitrogen,

127 Carlsbad, CA, USA).

128 Then libraries with different indices were multiplexed and loaded on an Illumina HiSeq

129 instrument according to manufacturer's instructions (Illumina, San Diego, CA, USA).

130 Sequencing was carried out using a 2x150 bp paired-end (PE) configuration. The raw data has

131 been deposited in NCBI with the following accession numbers: A35-2: SRR12283471,

132 SRR13090079, SRR13090078; A35-4: SRR12283470, SRR13090077, SRR13090076; A35-6:

133 SRR12283469; A7-2: SRR12283468, SRR13090082, SRR13090083; A7-4: SRR12283467,

134 SRR13090081, SRR13090080; A7-6: SRR12283466, SRR13100636, SRR13100635.

\section{RNA-seq data analysis}

136 The software of Trimmomatic v0.30 (http://usadellab.org/cms/index.php?page=trimmomatic)

137 was used to remove the technical sequences for the high quality clean data (Bolger et al., 2014).

138 As for mapping, the reference genome sequences were downloaded from Phytozome

139 (ftp://ftp.jgi-psf.org/pub/compgen/phytozome/v9.0/early_release/Gmax_275_Wm82.a2.v1/,

140 version Glyma 2.0, $975 \mathrm{Mb}$ ), and then the clean data were aligned to the reference genome using

141 Hisat2 (v2.0.1) (http://ccb.jhu.edu/software/hisat2/manual.shtml). Differential expression

142 analysis used the DESeq Bioconductor package, a model based on the negative binomial

143 distribution (http://www.bioconductor.org/packages/release/bioc/html/DESeq2.html) (Anders

144 and Huber, 2010, 2012). After controlling for the false discovery rate (Benjamini and Hochberg,

145 1995), the $P$-value threshold of $<0.05$ was used for detecting significant differential gene

146 expression. Gene Ontology (GO) and Kyoto Encyclopedia of Genes and Genomes (KEGG)

147 analyses were performed according to the methods described in Xin et al. (2018). 


\section{Principal component analysis}

150 Principal component analysis was performed to visualize sample-to-sample distances, as

151 described by Lee et al. (2014). This analysis was conducted in R (R Core Team, 2017).

152

153 Quantitative Real Time-PCR (qRT-PCR) analysis and statistical tests

154 The methods for qRT-PCR analysis and statistical tests were performed as described previously 155 (Zhao et al., 2018). The specific primers are shown in Table S1.

156

157 RESULTS

158 Phenotype identification and transcriptome sequencing

159 A7 and A35 are two cultivars of soybean from China. Their oil and protein contents in mature 160 seeds vary greatly, with the average protein content of A7 (50.8\%) being significantly higher 161 than in A35 (38.1\%), while the oil content in A7 (17.6\%) is much lower than in A35 (22.11\%)

162 (Figure 1B). To study the global changes in gene expression during seed development, seeds 163 from both cultivars were collected and analyzed by transcriptome sequencing at different 164 timepoints (weeks after flowering or WAF) resulting in the following samples: A7-2 WAF, A7-4 165 WAF, A7-6 WAF, A35-2 WAF, A35-4 WAF, A35-6 WAF (Figure 1A).

166 All the sequencing data are shown in Table 1. A total of 139 and 153 million clean reads were 167 acquired in A7 and A35, respectively. The reads were highly matched to the soybean reference 168 genome, and the statistical results showed that 54665387, 44537617, 40763561, 39120215, 16944375847 and 41099599 reads were mapped for A7-2, A7-4, A7-6, A35-2, A35-4 and A35-6, 170 respectively, with $90.3 \%$ average matching rate. The saturation and evenness of the

171 transcriptomes were further analyzed to estimate the sequencing depth and evenness. According 172 to the gene expression levels, the saturation curves were analyzed in four classifications: below $17325 \%, 25 \%-50 \%, 50 \%-75 \%$ and 75\%-100\% (Figure S1). The expression evenness was analyzed 174 through the whole transcripts from 5' to 3' (Figure S2). The results showed that the overall 175 quality of sequencing in this study was high and covered the vast majority of expressed genes. In 
176 addition, the correlation analysis of different samples showed that the average correlation of all

177 the comparison pairs was more than $90 \%$ (Figure S3).

\section{DEGs in paired comparisons of A7 and A35}

179 In order to detect significant DEGs, the $P$-value was adjusted as above, and the log2foldchange $>$ 1802 and $P$-value $<0.05$ were set as thresholds (Figure S4). In total, the DEGs from different 181 developmental stages and different soybean cultivars have been systematically compared and 182 valued. Comparing different time points (WAF) in the cultivar A7, 12638 DEGs were found in 183 A7-2-VS-A7-4 (7237 up-regulated and 5401 down-regulated); 7595 DEGs were found in A7-4184 VS-A7-6 (4196 up-regulated and 3399 down-regulated); and 17035 DEGs were found in A7-2185 VS-A7-6 (9736 up-regulated and 7299 down-regulated) (Figure 2A). Similarly, in A35 the 186 number of DEGs in A35-2-VS-A35-4 was 9364 (6134 up-regulated and 3230 down-regulated); 187 in A35-4-VS-A35-6 it was 7375 DEGs (4034 up-regulated and 3341 down-regulated); in A35-2188 VS-A35-6, it was 14231 DEGs (8828 up-regulated and 5403 down-regulated). Generally, the 189 DEGs in A7 and A35 mainly occurred in the 2- and 4- WAF pair and in the 2- and 6-WAF pair, 190 and many more up-regulated genes were observed than down-regulated genes, especially in the 191 early developmental stages (Figure 2). In seeds, the storage nutrients gradually accumulated from 192 the early stage of the seed development (2 WAF) to the late stage of seed development (6 WAF). 193 On this basis, we conclude that a large number of genes are activated mainly around 2 WAF to 194 meet the needs of energy and materials during the accumulation of storage nutrients in seeds.

195 Furthermore, the comparisons between A7 and A35 at the same stages were also performed. 196 Overall, 1312 DEGs (725 up and 587 down) were found in both A7 and A35 (Figure 2B). 197 However, at different developmental stages a divergence of the DEGs was observed. Firstly, 198 comparing A7 and A35, the DEGs mainly occurred in 2-WAF seeds, with the number of DEGs 199 in 2-WAF seeds (4818) being much higher than in the 4- (1371) and 6-WAF seeds (1515). This 200 observation was supported by the results of the principal component analysis (PCA) showing a 201 major difference between A7 and A35 at the early stage of seed development (2-WAF seeds) 202 (Figure S5). Secondly, in the 2-WAF seeds, the number of upregulated DEGs (3317) was 203 significantly higher than the number of downregulated genes (1501) in A35 compared to A7, 204 which indicated more genes were activated in A35 than A7 during the early developmental 205 stages of seeds. In addition, the time point specific DEGs were also compared, and the results 
206 were consistent with above ones, in which 3806, 446 and 708 specific DEGs have been 207 identified at $2 \mathrm{WAF}, 4 \mathrm{WAF}$ and $6 \mathrm{WAF}$, respectively.

\section{Analysis of DEG clustering and pathway enrichment}

209 To better understand the trends of DEG regulation during the seed development, hierarchical 210 clustering was performed and presented in a boxplot dendrogram (Figure S6). The dendrogram 211 provided a clear overview of the clade structure, in which the DEGs clustered to the up-regulated 212 or down-regulated clades, according to their expression trends. In the heatmap (Figure S6), 21325139 DEGs were shown, but only half of them (13262) were annotated in GO involving 214 biological process (5631), cellular component (1200) and molecular function (6431). 215 Furthermore, the DEGs concentrated in the functional groups such as catalytic activity, binding, 216 metabolic process, cellular process and biological regulation, which were consistent with the 217 processes of seed development including energy metabolism, nutrients accumulation and cell 218 proliferation (Figure 3A, Figure S7).

219 To further find out the involved metabolic pathways of the DEGs, the data were mapped against 220 the KEGG pathway database (Table S5). In A7, a total of 85 pathways with Q value $<0.05$ were 221 identified. Fifty eight, 46 and 33 pathways were mapped in the A7-2-VS-A7-4, A7-2-VS-A7-6 222 and A7-4-VS-A7-6 paired comparisons, respectively. In A35, 78 pathways were totally 223 identified: 46 pathways were in A35-2-VS-A35-4, 37 in A35-2-VS-A35-6 and 28 in A35-4-VS224 A35-6, respectively. Notably, 8 and 4 significantly mapped pathways were common to the three 225 paired comparisons in A7 and A35, respectively (Figure 3B). In A7, the common pathways 226 included ko05203 (Viral carcinogenesis), ko01524 (Platinum drug resistance), ko00073 (Cutin, 227 suberine and wax biosynthesis), ko05418 (Fluid shear stress and atherosclerosis), ko00480 228 (Glutathione metabolism), ko05034 (Alcoholism), ko04110 (Cell cycle), and ko04914 229 (Progesterone-mediated oocyte maturation), which were mainly involved in cell cycle and 230 stresses responses. However, in A35, the common pathways were ko00052 (Galactose 231 metabolism), ko00073 (Cutin, suberine and wax biosynthesis), ko00591 (Linoleic acid 232 metabolism) and ko00500 (Starch and sucrose metabolism), mainly involved in sugar and fatty 233 acids metabolism. Only one common pathway, namely ko00073, occurred in both A7 and A35, 234 which was related to the biosynthesis of cutin, suberine and wax in cell wall (Figure 3 ). When 235 specific developmental stages of A7 were compared with A35, lipid-related pathways (ko00592, 
236 ko00062, ko00071) and sugar-related pathways (ko00196, ko00195, ko00710, ko00010) were

237 found at the 2-WAF stage, while amino acid metabolism pathways (ko00360, ko00270) were

238 found at 4- and 6-WAF stages, respectively (Table S5). The divergence of the pathways in

239 different developmental stages of soybeans showed conformity with the differences in nutrient

240 contents between A7 and A35.

\section{Exploration of DEGs that may contribute to the oil and SSP contents in soybeans}

242 Given the significant difference in oil and protein contents in A7 and A35, the DEGs involved in

243 the synthetic and metabolic pathways of lipid, fatty acids, amino acids and proteins during the

244 seed development were specifically investigated (Figure 4). Firstly, the change trends of up- and

245 down-regulated DEGs involved in the lipid metabolism were V-shaped in A7 and A35 from 2-

246 VS-4, 4-VS-6 to 2-VS-6. Correlation analysis showed that the trends were consistent between

247 A7 and A35 (Figure 4A). Similarly, the change trends of DEGs related to the amino acid 248 metabolism were also V-shaped in A7 and A35 (Figure 4D). On the other hand, the change 249 trends of DEGs involved in fatty acid and protein metabolism were different in A7 and A35. As 250 for the fatty acids, the numbers of up- and down-regulated DEGs in A7 were similar in 2-VS-4 251 and 4-VS-6 pairs, but sharply decreased in 2-VS-6. However, in A35 the number of down252 regulated DEGs was increased linearly from 2-VS-4, 4-VS-6 to 2-VS-6, while the trend of the 253 up-regulated DEGs was V-shaped (Figure 4B). Notably, the trends of DEGs involved in protein 254 synthesis were opposite in A7 and A35. In A7, the number of up- and down-regulated DEGs was 255 first increased and then decreased, while in A35 it was the other way around (Figure 4C). 256 Notably, the divergent trends of numbers of DEGs involved in fatty acid and protein synthesis 257 were consistent with the difference in oil and protein contents in A7 and A35, which indicated 258 that these DEGs probably contribute to the content differences in A7 and A35.

259 To further reveal DEGs involved in oil and protein content differences in A7 and A35, the 260 significant DEGs were matched to the QTL database in SoyBase (https://www.soybase.org/). In 261 this way, two DEGs, Glyma.06G214800 and Glyma.07G034900, were found to be involved in 262 oil and linoleic acid contents. Glyma.06G214800 encodes 3-ketoacyl-CoA synthase (KCS) 263 catalyzing the elongation of C18 fatty acids while Glyma.07G034900 encodes linoleate 9S264 lipoxygenase (LOX1-5) which generates a peroxide by adding oxygen in the double bond of 265 linoleic acid. In our study, the expression levels of Glyma.06G214800 and Glyma.07G034900 
266 were down-regulated during the seed development in A35. However, the expression of

267 Glyma.06G214800 was not detected in A7, while the expression levels of Glyma.07G034900 in 268 A7 were significantly higher than in A35, which probably is related to the difference in oil 269 content between A7 and A35 (Figure 5A, Table S6).

270 No specific DEGs involved in protein content were matched to the QTL database. However, 271 previous studies have revealed that the expression of SSPs is mainly controlled by transcription 272 factors during the seed filling stages, the master regulators include LEC1, LEC2, ABI3, and 273 FUSCA3 (FUS3) (reviewed by Fedorova et al., 2002). In our study, the expression of LEC1s, $274 L E C 2$ s, FUS3s and $A B I 3$ s showed some divergence, in which the expression trends of LEC1s 275 (Glyma.07G268100 and Glyma.17G005600) and ABI3 (Glyma.02G099500, Glyma.01G087500, 276 and Glyma.10G204400) were basically consistent in A7 and A35, while LEC2s 277 (Glyma.20G035800 and Glyma.20G035700) and ABI3 (Glyma.20G186200) expression was 278 different (Figure 5, Table S6). Most of the consistently expressed transcription factors (TF) were 279 higher in A35 than A7 except for ABI3 (Glyma.02G099500 and Glyma.01G087500) (Figure 5, 280 Table S6). Considering the positive relationship of TF expression with the content of SSPs, the 281 two DEGs, ABI3s (Glyma.02G099500 and Glyma.01G087500) and LEC2s (Glyma.20G035800 282 and Glyma.20G035700), might be correlated with the difference of SSPs between A7 and A35. 283 Furthermore, a sugar transporter, SWEET10a (Glyma.15G049200), contributing to the increase 284 in seed weight and oil content and reduced protein content was identified by the QTL database 285 search. Its expression patterns were different in A7 and A35, with an inverted V shape in A7 and 286 linear decline in A35. The biggest divergence between A7 and A35 occurred at the 2-WAF stage, 287 where the expression in A35 is much higher than in A7 ( $p<0.001$, Figure 5, Table S6).

\section{Expression flux in the Fatty acids synthesis pathway}

289 The pathways of fatty acid metabolism mainly include fatty acid biosynthesis (ko00061), fatty 290 acid elongation (ko00062) and fatty acid degradation (ko00071) (Figure 6, Figure S8). All the 291 DEGs and their expression trends in these pathways have been marked in Figure 6 to identify 292 their expression flux. The highest expression levels in the whole pathway were observed for fatty 293 acid dehydrogenase 2 (FAD2s) encoded by Glyma.10G278000 and Glyma.20G111000. Both 294 DEGs were specifically expressed in seeds and the expression levels were increased during the 295 seed development. The expression levels of FAD2s were higher in A35-2WAF than in A7- 
296 2WAF, which suggested that FAD2 genes might be essential for the content of oil in A7 and A35.

297 Notably, the expression levels of key enzymes of the fatty acid elongation pathway were 298 drastically reduced during the development of seeds in A7 and A35 (Figure 6) and their 299 expression levels in A35 were lower than in A7. The results might explain why the fatty acids in 300 soybean seeds were mainly present in the form of C18. As for fatty acid degradation, the 301 expression of enoyl-CoA hydratase/3-hydroxyacyl-CoA dehydrogenase (MFP2/HAD) and 302 acetyl-CoA C-acetyltransferase (atoB) was stable in the development of seeds in A7 and A35 303 (Figure 6).

\section{The qRT-PCR analysis of DEGs in soybeans}

305 To confirm the RNA-seq results in our research, we selected 6 significant DEGs related to the 306 fatty acid metabolism and the synthesis of amino acids for qRT-PCR analysis (Figure 7, Table 307 S6). In all, the expression results of qRT-PCR were consistent with the RNA-seq results. Among 308 them, the expression of Glyma.13G035200 was significantly increased along the seed 309 development, while Glyma.16G147300 was gradually decreased. The expression trend of 310 Glyma.06G211300 was decreased from 2-WAF to 4-WAF seeds, and then was increased in 6311 WAF seeds. The expression trends of Glyma.17G047000, Glyma.17G027600 and 312 Glyma.06G183900, on the other hand, were bell-shaped, increasing from 2-WAF to 4-WAF 313 seeds and then decreased in 6-WAF seeds. The results verified that our RNA-seq data were 314 reliable.

\section{DISCUSSION}

317 It has been shown that seeds of different soybean cultivars vary widely in nutrient content with a 318 negative correlation between oil and protein contents (Chuang et al., 2003). In our study, the two 319 soybean cultivars A7 and A35, with similar genetical background, showed distinctive differences 320 in storage protein and oil contents, providing a useful system for comparative studies of the 321 regulation of seed nutrient accumulation. Our transcriptome data of three stages of seed 322 development, 2-, 4-, and 6-WAF seeds, were used to analyze and compare gene expression 323 trends. Similar to previous studies (Bao and Ohlrogge, 1999), there were more DEGs at the early 324 stage of seed development with up-regulated DEGs more numerous than down-regulated ones in 
325 both soybean cultivars (Figure 2A). The accumulation of nutrients, especially oils and SSPs, is 326 essential to seed development. The accumulation of nutrients is a complex and dynamic process, 327 which is influenced by multiple genetic and environmental factors (Gupta et al., 2017; Hills, 328 2006). Until now, studies including genetics, omics, QTL and GWAS have been performed to 329 investigate the mechanisms of seed filling (Hyten et al., 2004a,b; Li et al., 2018a,b; Gupta et al., 330 2017; Agrawal et al., 2008). It has been revealed that the oil content of soybean seeds gradually 331 increased until 40 days after flowering (DAF), and stayed steady in later stages (Li et al., 2015, 332 Collakova et al., 2013), which is consistent with our results. In our study, the comparison of 333 transcriptome data from different developmental stages of seeds and different soybean cultivars 334 revealed that the change trends of DEGs involved in the synthesis of fatty acids were basically 335 consistent in A7 and A35, including the down-regulation of fatty acid synthesis in 6-WAF seeds. 336 It has been reported that the supply of fatty acids was the limiting factor for the accumulation of 337 oil in embryos (Bao and Ohlrogge, 1999). Linoleic acid is the main fatty acid of soybean oil. 338 FAD2 is essential to convert oleic acid into linoleic acid. Notably, FAD2 genes involved in the 339 fatty acid pathways were highly expressed in all periods, and in A35 it was obviously higher than 340 in A7, which indicated that the oil difference in A35 and A7 might be attributed to the expression 341 difference of FAD2 genes. In our previous study, it was also shown that the bioactivity of FAD2 342 was correlated with oil content in different plants (Zhao et al., 2019). Therefore, the oil content 343 of seeds might be controlled by both the expression and bioactivity of FAD2. Furthermore, the 344 DEGs in the fatty acid degradation pathway were similar in A7 and A35, and the result showed 345 that the degradation pathway was not the main reason for divergence in the two cultivars. 346 Furthermore, we analyzed the fatty acid elongation pathway and found that the expression trends 347 were drastically reduced during the seed development in A7 and A35 (Figure 5,6). Moreover, the 348 expression levels of KCS in A35 were lower than in A7. The results indicated that more fatty 349 acids were present in the form of C18 in the late development of seeds in A35, which was 350 consistent with the composition of soybean oil. Based on the above results, it can be concluded 351 that the differences in gene expression related to biosynthesis and elongation of fatty acids 352 during the seed development contributed to the difference of oil content in A35 and A7. More 353 experiments need to be done for more details.

354 In addition, the content of proteins was also significantly different in A7 and A35. The change 355 trends of DEGs involved in the proteins synthesis were different, especially in 4-VS-6 of A7 and 
356 A35 (Figure 4C), which suggested the content differences between A7 and A35 might be due to 357 the expression levels of the genes encoding proteins between 4 WAF seeds and 6 WAF seeds. 358 Unfortunately, in our study, we didn't find the identified protein DEGs which matched into the 359 QTL database. The possible reason might be that protein content is a complex quality trait and is 360 influenced by many different genes. TFs are essential to regulate the gene expression. Previous 361 studies have revealed that the expression of SSPs is mainly controlled by TFs, LEC1, LEC2, $362 A B I 3$, and FUSCA3 (FUS3), during the seed filling stages (reviewed by Verdier and Thompson, 363 2008). These TFs interacted in a network in which LEC1 induces the expression of $L E C 2, A B I 3$ 364 and FUS3. Moreover, LEC1 and LEC2 genes regulate each other, and activate the expression of 365 FUS3 and ABI3, and then activate the SSP genes (Fedorova et al., 2002; Kagaya et al., 2005; 366 Santos Mendoza et al., 2005; Keith et al., 1994; Parcy et al., 1997; To et al., 2006; Braybrook et 367 al., 2006). In our study, the expression of LEC1s, LEC2s, FUS3s and ABI3s showed some 368 divergence, in which the expression trends of LEC1s (Glyma.07G268100 and 369 Glyma.17G005600), ABI3 (Glyma.02G099500, Glyma.01G087500, and Glyma.10G204400) 370 were basically consistent in A7 and A35, while LEC2s (Glyma.20G035800 and 371 Glyma.20G035700) and ABI3 (Glyma.20G186200) showed different trends (Figure 5, Table S6). 372 Besides, most of consistently expressed TFs were higher in A35 than A7 except for $A B I 3$ 373 (Glyma.02G099500 and Glyma.01G087500) (Figure 5, Table S6). Considering the positive 374 relationship of TF expression and content of SSPs, we suggest that the two DEGs, $A B I 3 \mathrm{~s}$ 375 (Glyma.02G099500 and Glyma.01G087500) and LEC2s (Glyma.20G035800 and 376 Glyma.20G035700), are correlated with the difference of SSPs between A7 and A35. In addition, 377 an important sugar transporter, SWEET10a, has been identified to be involved in the seed size, 378 and oil and protein contents (Wang et al., 2020), and in our study very similar results were 379 observed. Note that our results further indicated that the difference in expression patterns and 380 levels of SWEET10a at the early stage of seed development might be related to the different 381 protein and oil contents of soybean seeds. More research will be needed to confirm this 382 relationship.

383 It has been reported that the synthesis of special amino acids, such as methionine and asparagine, 384 is also related to the accumulation of proteins (Li et al., 2015; Galili et al., 2016; Molvig et al., 385 1997; Zhao et al., 2018). It has also been reported that asparagine was the major form of nitrogen 386 imported into seeds from the vegetative organs. The asparaginase enzyme (Glyma.05G018300) 
387 was predicted to interconvert amino acids for protein synthesis during seed filling (Li et al., 388 2015). In our study, the expression of Glyma.05G018300 was also analyzed, but no significant 389 differences were found in any comparison indicating the gene might be not involved in the 390 protein differences in the studied cultivars (Figure S9).

\section{CONCLUSIONS}

392 In this study, comparative transcriptome analysis was performed to study the dynamic gene 393 expression at different developmental stages of soybean seeds in the A7 and A35 cultivars. The 394 results showed that more DEGs occurred at early stages of seed development, especially at the 2 395 WAF stage, and there were more up-regulated DEGs at the early stages compared with the late 396 stages. The change trends of the DEGs involved in metabolism of nutrients such as oil and SSPs 397 were different. The results indicate that DEGs related to the synthesis and elongation of fatty 398 acids, FAD2, KCS and LOX, contribute to the oil content difference in A7 and A35. The data 399 also suggest that the accumulation of SSPs is controlled by transcription regulators such as $A B I 3$ 400 and $L E C 2$, while the sugar transporter SWEET10a might contribute to both oil and SSP contents.

401

402

ADDITIONAL INFORMATION AND DECLARATIONS

403

\section{Funding}

404 This work was supported by Zhejiang Provincial Major Agriculture Science and Technology

405 Special Sub-project (Grant No. 2016C02050-10-3).

\section{Competing Interests}

407 The authors declare no competing financial interest.

\section{Authors' contributions}

409 M.Z. and L.P. conceived and designed the analyses. L. Q., M. W. and H.C. performed most of

410 the experiments and carried out most of the analysis. X. S. and W. L. performed RT-PCR

411 expression analyses. M. Z. and L.P. analyzed and interpreted the data. F. Y. and Z.W.

412 coordinated the work. M.Z. drafted the manuscript. All authors have read and approved the final 413 manuscript.

414

\section{SUPPLEMENTARY MATERIALS}


416 Figure S1. The saturation curves of all the samples in A7 and A35.

417 Figure S2. The evenness curves of samples in A7 and A35.

418 Figure S3. The correlation analysis of samples in A7 and A35.

419 Figure S4. Volcano plots of DEGs. The up-regulated and down-regulated DEGs are shown in 420 red and blue, respectively. The x-axis represents the fold change of DEGs in different samples.

421 The y-axis represents the statistical significance of gene expression differences.

422 Figure S5. The principal component analysis (PCA) analysis of samples in A7 and A35.

423 Figure S6. Clustering analysis of the DEGs. The tree was constructed with $\log 10(\mathrm{RPKM}+1)$.

424 Blue, white and red indicate high, intermediate and low expression, respectively. The clustered 425 genes are shown in the different color such as green, light blue and purple. 2 WAF, 4 WAF and 6 426 WAF seeds were labeled with A7-2,-4,-6 and A35-2, -4, -6. Each sample has three repetitions 427 labeled $a, b$ and $c$.

428 Figure S7. GO analysis of the DEGs. A1-A4: the enriched GO terms in A35-2-VS-A7-2, A35-4429 VS-A7-4, A35-6-VS-A7-6 and A35-VS-A7. B: the DEG numbers in different pairwise 430 comparisons. The functions related to molecular function, cellular component and biological 431 process are shown in red, green and blue, respectively.

432 Figure S8. The fatty acid and amino acid metabolism pathways in the KEGG database.

433 Figure S9. The expression trends of the asparaginase enzyme family in different pairwise 434 comparisons. The Neighbor joining tree was constructed with amino acid sequences. The red, 435 green and gray boxes represent their expression level for down-regulated, up-regulated and not 436 changed, respectively.

437 Table S1. The primers of DEGs used in qRT-PCR.

438 Table S2. The overview of DEGs in A7-2-VS-A7-4, A7-2-VS-A7-6 and A7-4-VS-A7-6.

439 Table S3. The overview of DEGs in A35-2-VS-A35-4, A35-2-VS-A35-6 and A35-4-VS-A35-6

440 Table S4. The overview of DEGs in A35-2-VS-A7-2, A35-4-VS-A7-4, A35-6-VS-A7-6 and 441 A35-VS-A7.

442 Table S5. The significant KEGG pathways in different compared pairs.

443 Table S6. The expression levels of qRT-PCR, the protein and oil content in A7 and A35, and 444 statistical significance.

445

446

Peer] reviewing PDF | (2020:07:50770:3:0:NEW 15 Dec 2020) 
447 REFERENCES

448 Graham PH, Vance CP. 2003. Legumes: importance and constraints to greater use. Plant 449 Physiology 131:872-877.

450 Hurburgh CR. 1994. Long-term soybean composition patterns and their effect on processing. 451 Journal of the American Oil Chemists Society 71:1425-1427.

452 Harada JJ, Barker SJ, Goldberg RB. 1989. Soybean beta-conglycinin genes are clustered in 453 several DNA regions and are regulated by transcriptional and posttranscriptional processes. the 454 Plant Cell 1:415-425.

455 Nielsen NC, Dickinson CD, Cho TJ, Thanh VH, Scallon BJ, Fischer RL, Sims TL, Drews 456 GN, Goldberg RB. 1989. Characterization of the glycinin gene family in soybean. the Plant Cell $457 \quad 1: 313-328$.

458 Meinke D, Chen J, Beachy R. 1981. Expression of storage-protein genes during soybean seed 459 development. Planta 153:130-139.

460 Hyten DL, Pantalone VR, Sams CE, Saxton AM, Landau-Ellis D, Stefaniak TR, Schmidt 461 ME. 2004a. Seed quality QTL in a prominent soybean population. Theoretical and Applied 462 Genetics 109:552-561.

463 Hyten DL, Pantalone VR, Saxton AM, Schmidt ME, Sams CE. 2004b. Molecular mapping 464 and identification of soybean fatty acid modifier quantitative trait loci. Journal of the American 465 Oil Chemists Society 12:1115-1118.

466 Li D, Zhao X, Han Y, Li W, Xie F. 2018a. Genome-wide association mapping for seed protein 467 and oil contents using a large panel of soybean accessions. Genomics 111(1):90-95.

468 Li Y, Reif JC, Hong H, Li H, Liu Z, Ma Y, Li J, Tian Y, Li Y, Li W. 2018b. Genome-wide 469 association mapping of QTL underlying seed oil and protein contents of a diverse panel of 470 soybean accessions. Plant Science 266:95-101.

471 Wilcox JR, Shibles RM. 2001. Interrelationships among seed quality attributes in soybean.

472 Crop Science 41:11-14.

473 Gupta M, Bhaskar PB, Sriram S, Wang PH. 2017. Integration of omics approaches to 474 understand oil/protein content during seed development in oilseed crops. Plant Cell Reports 475 36:637-652.

476 Le BH, Wagmaister JA, Kawashima T, Bui AQ, Harada JJ, Goldberg RB. 2007. Using 477 genomics to study legume seed development. Plant Physiology 144:562-574. 
478 Akond M, Liu S, Boney M, Kantartzi SK, Meksem K, Bellaloui N, Lightfoot DA, Kassem

479 MA. 2014. Identification of quantitative trait loci (QTL) underlying protein, oil, and five major

480 fatty acids' contents in soybean. American Journal of Plant Sciences 5:158-167.

481 Lee JD, Bilycu KD, Shannon JG. 2007. Genetics and breeding for modified fatty acid profile in 482 soybean seed oil. Journal of Crop Science and Biotechnology 10:201-210.

483 Hwang EY, Song Q, Jia G, Specht JE, Hyten DL, Costa J, Cregan PB. 2014. A genome-wide 484 association study of seed protein and oil content in soybean. BMC Genomics 15:1.

485 Hills MJ. 2004. Control of storage-product synthesis in seeds. Current Opinion in Plant Biology $4867: 302-308$.

487 Korte A, Farlow A. 2013. The advantages and limitations of trait analysis with GWAS: a 488 review. Plant Methods 9:29.

489 Panthee DR, Pantalone VR, Saxton AM. 2006. Modifier QTL for fatty acid composition in 490 soybean oil. Euphytica 152:67-73.

491 Phansak P, Soonsuwon W, Hyten DL, Song Q, Cregan PB, Graef GL, Specht JE. 2016.

492 Multi-population selective genotyping to identify soybean (Glycine max (L.) Merr.) seed protein 493 and oil QTLs. G3-Genes Genomes Genetics 6(6):1635-1648.

494 Wang X, Jiang GL, Green M, Scott RA, Hyten DL. 2014. Quantitative trait locus analysis of 495 unsaturated fatty acids in a recombinant inbred population of soybean. Molecular Breeding 496 33:281-296.

497 Wang S, Liu S, Wang J, Yokosho K, Zhou B, Yu YC, Liu Z, Frommer WB, Ma JF, Chen

498 LQ. 2020. Simultaneous changes in seed size, oil content, and protein content driven by 499 selection of SWEET homologues during soybean domestication. National Science Review 500 nwaa110. DOI: $10.1093 /$ nsr/nwaa1 10.

501 Li L, Hur M, Lee JY, Zhou W, Song Z, Ransom N, Demirkale CY, Nettleton DS, Westgate 502 ME, Arendsee ZW. 2015. A systems biology approach toward understanding seed composition 503 in soybean. BMC Genomics 16(3):S9.

504 Ruuska SA, Girke T, Benning C, Ohlrogge JB. 2002. Contrapuntal networks of gene 505 expression during Arabidopsis seed filling. the Plant Cell 14(6):1191-1206.

506 Palovaara J, Saiga S, Weijers D. 2013. Transcriptomics approaches in the early Arabidopsis 507 embryo. Trends in Plant Science 18(9):514-521.

508 Fait A, Angelovici R, Less H, Ohad I, Urbanczyk-Wochniak E, Fernie AR, Galili G. 2006. 
509 Arabidopsis seed development and germination is associated with temporally distinct metabolic 510 switches. Plant physiology 142(3):839-854.

511 Fedorova M, van de Mortel J, Matsumoto PA, Cho J, Town CD, VandenBosch KA, Gantt

512 JS, Vance CP. 2002. Genome-wide identification of nodule-specific transcripts in the model

513 legume Medicago truncatula. Plant Physiology 130(2):519-537.

514 Gallardo K, Firnhaber C, Zuber H, Hericher D, Belghazi M, Henry C, Kuster H,

515 Thompson R. 2007. A combined proteome and transcriptome analysis of developing Medicago

516 truncatula seeds: evidence for metabolic specialization of maternal and filial tissues. Molecular

517 \& cellular proteomics 6(12):2165-2179.

518 Li F, Wu X, Tsang E, Cutler AJ. 2005. Transcriptional profiling of imbibed Brassica napus 519 seed. Genomics 86(6): 718-730.

520 Lan L, Chen W, Lai Y, Suo J, Kong Z, Li C, Lu Y, Zhang Y, Zhao X, Zhang X, Zhao X,

521 Zhang X. 2004. Monitoring of gene expression profiles and isolation of candidate genes

522 involved in pollination and fertilization in rice (Oryza sativa L.) with a 10K cDNA microarray.

523 Plant Molecular Biology 54(4):471-487.

524 Furutani I, Sukegawa S, Kyozuka J. 2006. Genome-wide analysis of spatial and temporal gene

525 expression in rice panicle development. Plant Journal 46(3):503-511.

526 Watson L, Henry RJ. 2005. Microarray analysis of gene expression in germinating barley

527 embryos (Hordeum vulgare L.). Functional \& Integrative Genomics 5(3): 155-162.

528 Collakova E, Aghamirzaie D, Fang Y, Klumas C, Tabataba F, Kakumanu A, Myers E,

529 Heath LS, Grene R. 2013. Metabolic and transcriptional reprogramming in developing soybean

530 (Glycine max) embryos. Metabolites 3(2):347-372.

531 Libault M, Farmer A, Joshi T, Takahashi K, Langley RJ, Franklin LD, He J, Xu D, May G,

532 Stacey G. 2010. An integrated transcriptome atlas of the crop model Glycine max, and its use in

533 comparative analyses in plants. Plant Journal 63:86-99.

534 Severin AJ, Woody JL, Bolon YT, Joseph B, Diers BW, Farmer AD, Muehlbauer GJ,

535 Nelson RT, Grant D, Specht JE. 2010. RNA-Seq Atlas of Glycine max: a guide to the soybean

536 transcriptome. BMC Plant Biology 10:160.

537 Verdier J, Thompson RD. 2008. Transcriptional regulation of storage protein synthesis during 538 dicotyledon seed filling. Plant Cell Physiology 49(9):1263-1271.

539 O’Rourke JA, Bolon YT, Bucciarelli B, Vance CP. 2014. Legume genomics: understanding 
540 biology through DNA and RNA sequencing. Annals of Botany 113:1107-1120.

541 Zhao M, Wang W, Wei L, Chen P, Yuan F, Wang Z, Ying X. 2018. Molecular evolution and

542 expression divergence of three key Met biosynthetic genes in plants: CGS, HMT and MMT.

543 PeerJ 6.

544 Liu X, Qin D, Piersanti A, Zhang Q, Miceli C, Wang P. 2020. Genome-wide association study

545 identifies candidate genes related to oleic acid content in soybean seeds. BMC Plant Biology

546 20(1):1-14.

547 Chung J, Babka HL, Graef GL, Staswick PE, Lee DJ, Cregan PB, Shoemaker RC, Specht

548 JE. 2003. The seed protein, oil, and yield QTL on soybean linkage group I. Crop Science

549 43(3):1053-1067.

550 Agrawal GK, Hajduch M, Graham K, Thelen JJ. 2008. In-depth investigation of the soybean

551 seed-filling proteome and comparison with a parallel study of rapeseed. Plant Physiology

552 148:504-518.

553 Bao X, Ohlrogge J. 1999. Supply of fatty acid is one limiting factor in the accumulation of

554 triacylglycerol in developing embryos. Plant Physiology 120 (4): 1057-1062.

555 Kagaya Y, Toyoshima R, Okuda R, Usui H, Yamamoto A, Hattori T. 2005. LEAFY

556 COTYLEDON1 controls seed storage protein genes through its regulation of FUSCA3 and

557 ABSCISIC ACID INSENSITIVE3. Plant and Cell Physiology 46:399-406.

558 Santos Mendoza M, Dubreucq B, Miquel M, Caboche M, Lepiniec L. 2005. LEAFY

559 COTYLEDON 2 activation is sufficient to trigger the accumulation of oil and seed specific

560 mRNAs in Arabidopsis leaves. FEBS Letters 579:4666-4670.

561 Keith K, Kraml M, Dengler NG, McCourt P. 1994. fusca3: a heterochronic mutation affecting

562 late embryo development in Arabidopsis. the Plant Cell 6:589-600.

563 Parcy F, Valon C, Kohara A, Mise' ra S, Giraudat J. 1997. The ABSCISIC ACID-

564 INSENSITIVE3, FUSCA3, and LEAFY COTYLEDON1 loci act in concert to control multiple

565 aspects of Arabidopsis seed development. the Plant Cell. 9:1265-1277.

566 To A, Valon C, Savino G, Guilleminot J, Devic M, Giraudat J, Parcy F. 2006. A network of

567 local and redundant gene regulation governs Arabidopsis seed maturation. the Plant Cell.

568 18:1642-1651.

569 Braybrook SA, Stone SL, Park S, Bui AQ, Le BH, Fischer RL, Goldberg RB, Harada JJ.

570 2006. Genes directly regulated by LEAFY COTYLEDON2 provide insight into the control of 
571 embryo maturation and somatic embryogenesis. Proceedings of the National Academy of 572 Sciences USA 103:3468-3473.

573 Galili G, Amir R, Fernie AR. 2016. The regulation of essential amino acid synthesis and 574 accumulation in plants. Annual Review of Plant Biology 67:153-178.

575 Molvig L, Tabe LM, Eggum BO, Moore AE, Craig S, Spencer D, Higgins TJV. 1997.

576 Enhanced methionine levels and increased nutritive value of seeds of transgenic lupins (Lupinus

577 angustifolius L.) expressing a sunflower seed albumin gene. Proceedings of the National 578 Academy of Sciences USA 94:8393-8398.

579 Zhao M, Chen P, Wang W, Yuan F, Zhu D, Wang Z, Ying X. 2018. Molecular evolution and 580 expression divergence of HMT gene family in plants. International Journal of Molecular 581 Sciences 19:1248.

582 Dong XL, Bai PL, Wang JM, Ruan CJ. 2011. Comparative study on determination of seed oil 583 content of energy plants by using NMR and Soxhlet Extraction. Renewable Energy 29(3):21-24.

584 Bolger AM, Lohse M, Usadel B. 2014. Trimmer for Illumina sequence data. Bioinformatics 585 btu170.

586 Anders S, Huber W. 2010. Differential expression analysis for sequence count data. Genome 587 Biology 11:R106.

588 Anders S, Huber W. 2012. Differential expression of RNA-Seq data at the gene level-the 589 DESeq package. EMBL.

590 Benjamini Y, Hochberg Y. 1995. Controlling the false discovery rate: a practical and 591 powerful approach to multiple testing. Journal of the Royal Statistical Society: Series B $592 \quad$ 57:289-300.

593 Xin Y, Li C, Guo Y, Xiao R, Zhang H, Zhou G. 2019. RNA-Seq analysis reveals a negative 594 role of MSMO1 with a synergized NSDHL expression during adipogenesis of 3T3-L1.

595 Bioscience, Biotechnology, and Biochemistry 83(4):641-652.

596 R Core Team. 2017. R: A language and environment for statistical computing. R Foundation for 597 Statistical Computing, Vienna, Austria. URL https://www.R-project.org/.

598 Lee KJ, Yin W, Arafat D, Tang Y, Uppal, K., Tran V, Cabrera-Mora M, Lapp SA, Moreno 599 A, Meyer E. 2014. Comparative transcriptomics and metabolomics in a rhesus macaque drug 600 administration study. Frontiers in Cell and Developmental Biology 2:54-54.

601 Livak KJ, Schmittgen TD. 2001. Analysis of relative gene expression data using real-time 
602 quantitative PCR and the 2- $\Delta \Delta \mathrm{Ct}$ method. Methods. 25:402-408.

603 Zhao M, Wang W, Wei L, Chen P, Peng L, Qin Z, Yuan F, Wang Z, Ying X. 2019. The

604 evolution and biocatalysis of FAD2 indicate its correlation to the content of seed oil in plants.

605 International Journal of Molecular Sciences 20(4):849. 


\section{Figure 1}

Figure 1. Seed phenotypes, and the protein and oil content in A7 and A35

Figure 1. Seed phenotypes, and the protein and oil content in A7 and A35. A. Phenotypes of 2-WAF, 4-WAF and 6-WAF seeds. B. The total protein and oil content in the mature seeds of A7 and A35. X-axis represented the types of protein and oil. Y-axis represented the dry seeds oil and protein percentage. Error bar: standard deviation. The significance was tested in comparison with the contents of oil and protein in A7 (blue columns). The * and ** represent the significance at a $P<0.05$ and $P<0.01$ levels, respectively. 


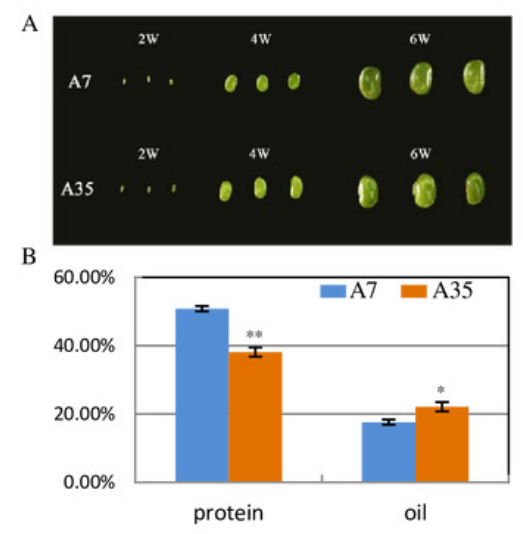




\section{Figure 2}

Figure 2. The gene numbers of significantly different expression among samples.

The $x$-axis represented the comparison pairs. The $y$-axis represented the number of DEGs. A. The comparison between 2-, 4-, 6-WAF in cultivars. B. The comparison between A7 and A35. The red and blue lines represent up-regulated and down-regulated DEGs, respectively. 

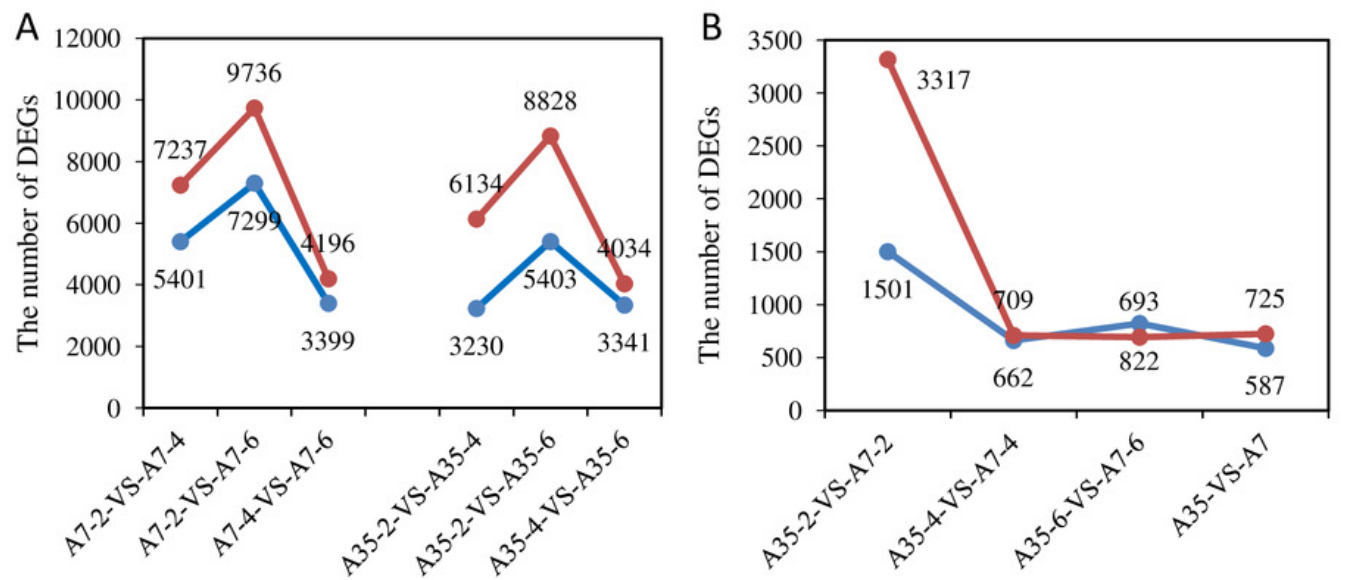


\section{Figure 3}

Figure 3. GO analysis and Pathways enrichment of DEGs in three paired comparisons in A7 and A35.

The functions related to molecular function, cellular component and biological process were shown in red, green and blue, respectively. The numbers of significantly rich pathways were marked on the map, respectively. 

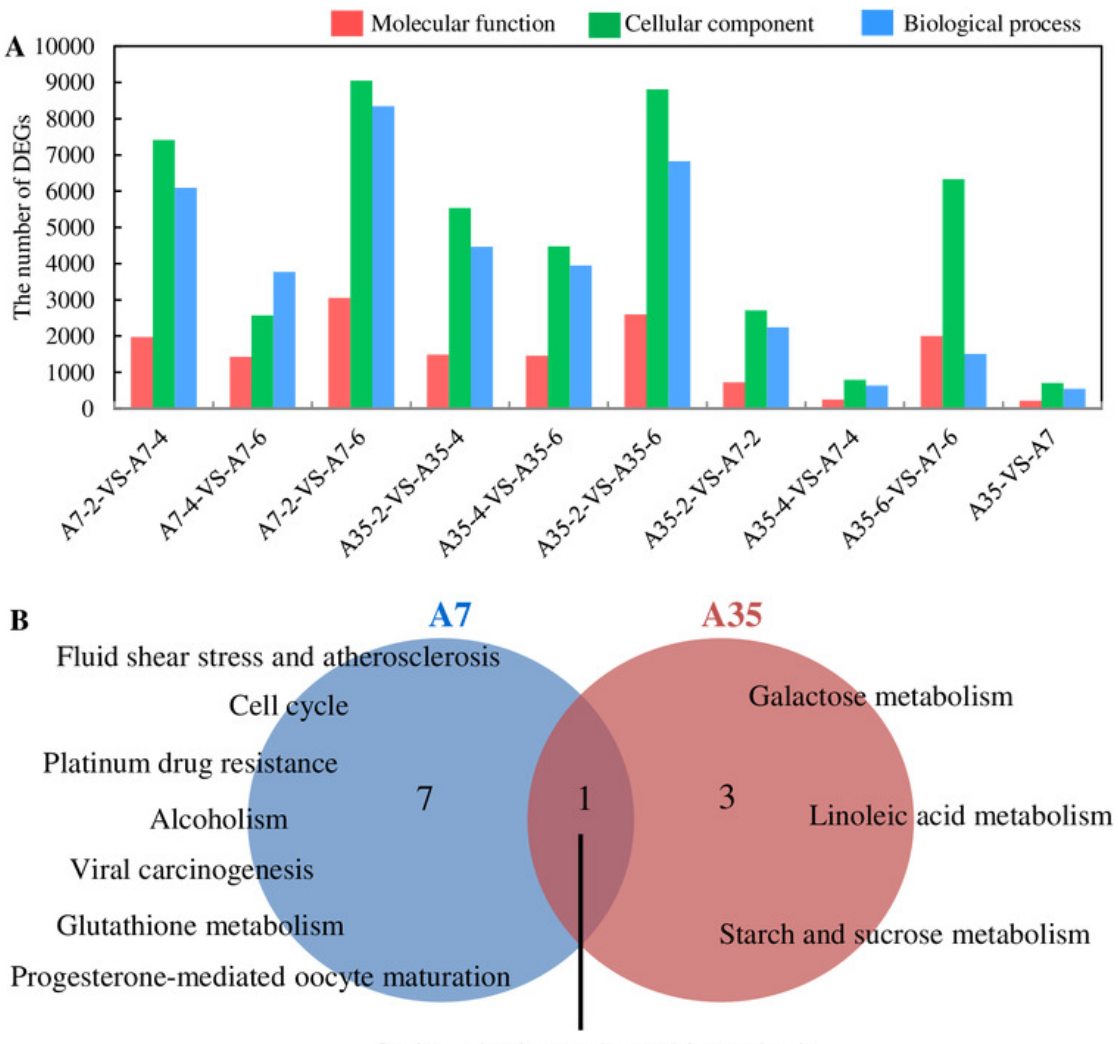

Cutin, suberine and wax biosynthesis 


\section{Figure 4}

Figure 4. The analysis of the DEGs numbers involved in oil and protein metabolism.

A-D: the numbers of DEGs in lipid, fatty acids, proteins and amino acids metabolism in A7 and $A 35$, respectively. The $x$-axis represented the comparison pairs. The $y$-axis represented the number of DEGs. The white and gray backgrounds represented the down- and upregulated DEGs, respectively. 

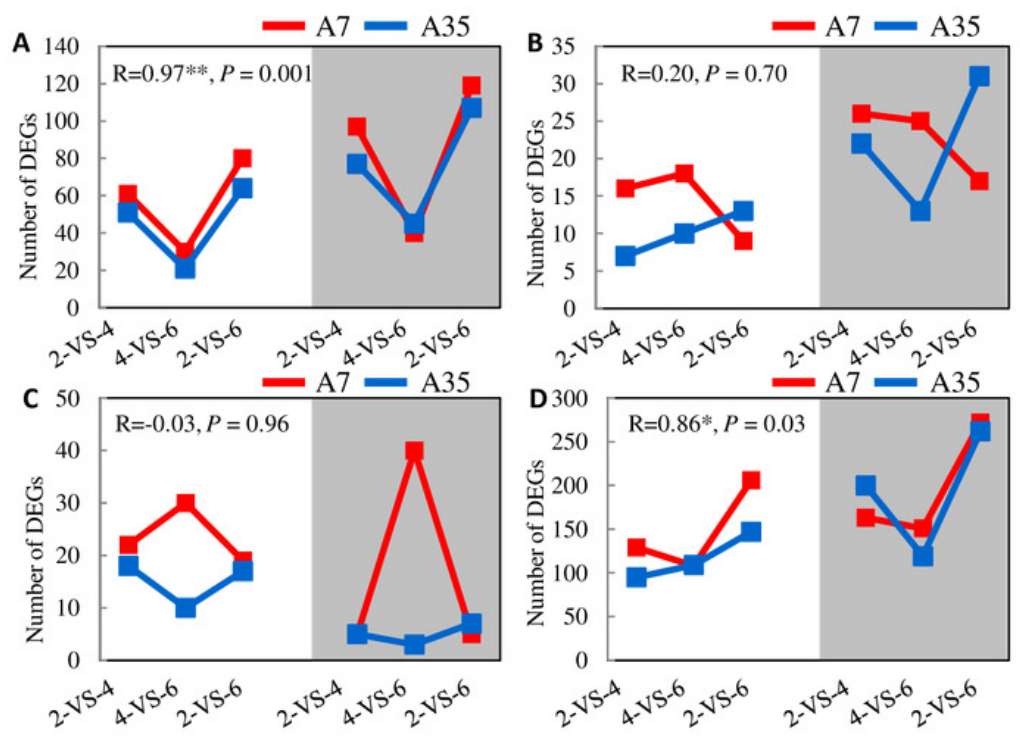
Figure 5

Figure 5. The expression trends of DEGs related to oil and SSPs.

$\mathrm{X}$-axis represented the developmental stages; Y-axis represented FPKM in RNA-Seq. 

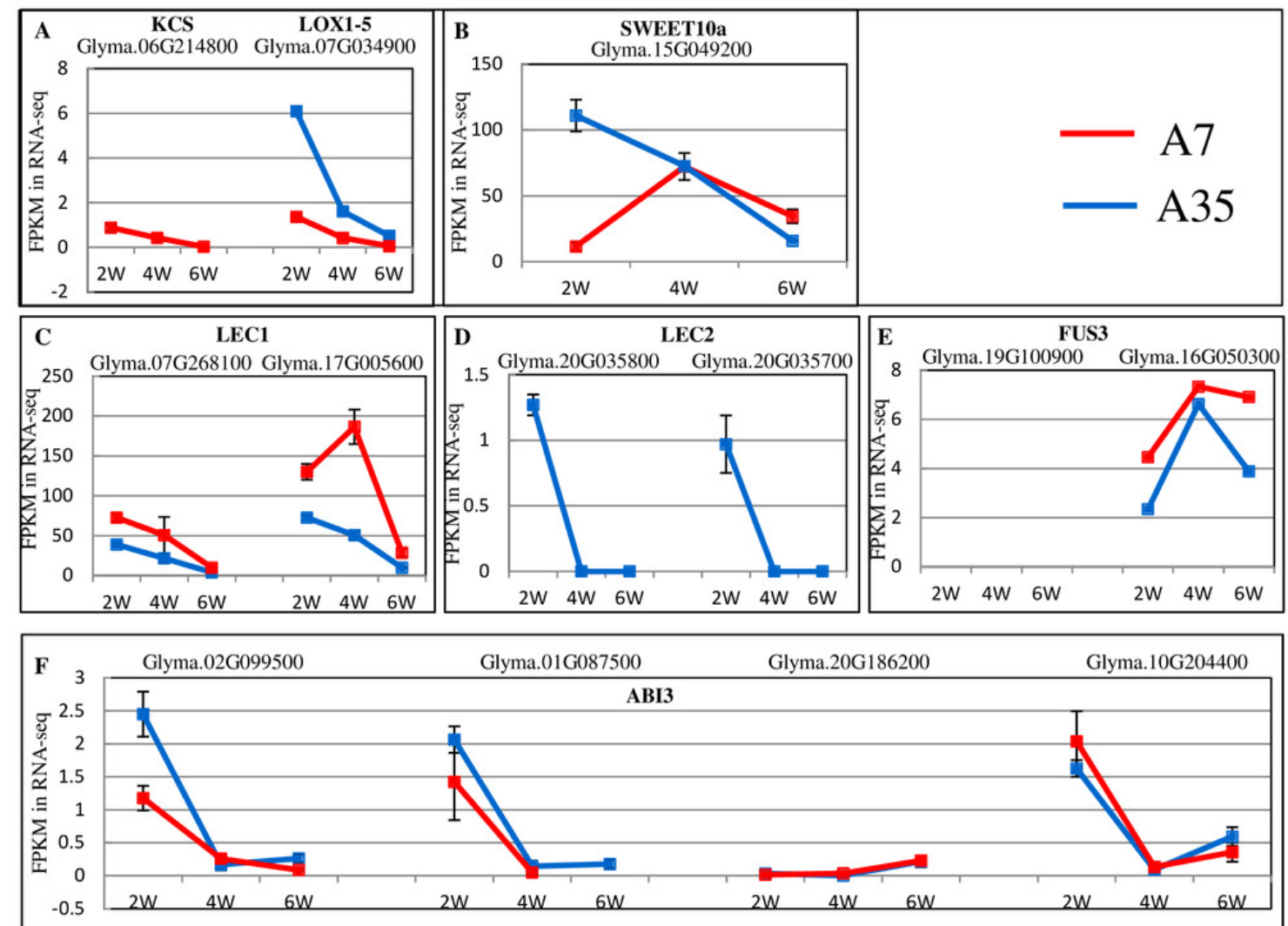


\section{Figure 6}

Figure 6. DEGs in soybeans associated with the fatty acid biosynthetic, elongation and degradation pathways.

Each square represents a comparative pair. The squares from left to right represent: A7-2, A7-4, A7-6, A35-2, A35-4 and A35-6. The red, blue squares indicate their expression levels. The enzymes are marked in blue in pathways. ACCase: acetyl-CoA carboxylase; MAT: malonyl-CoA; ACP transacylase; ACP: acyl carrier protein; FAS: fatty acid synthases; FATA/B: oleoyl-[acyl-carrier-protein] hydrolase; FAD2: fatty acid desaturase 2; ACOX: acyl-CoA oxidase; MFP2: enoyl-CoA hydratase; HAD: 3-hydroxyacyl-CoA dehydrogenase; atoB: acetylCoA C-acetyltransferase; KCS: 3-ketoacyl-CoA synthase; KAR: very-long-chain 3-oxoacyl-CoA reductase; PHS1: very-long-chain (3R)-3-hydroxyacyl-CoA dehydratase; TER: very-long-chain enoyl-CoA reductase; ACOT: acyI-CoA thioesterase. 


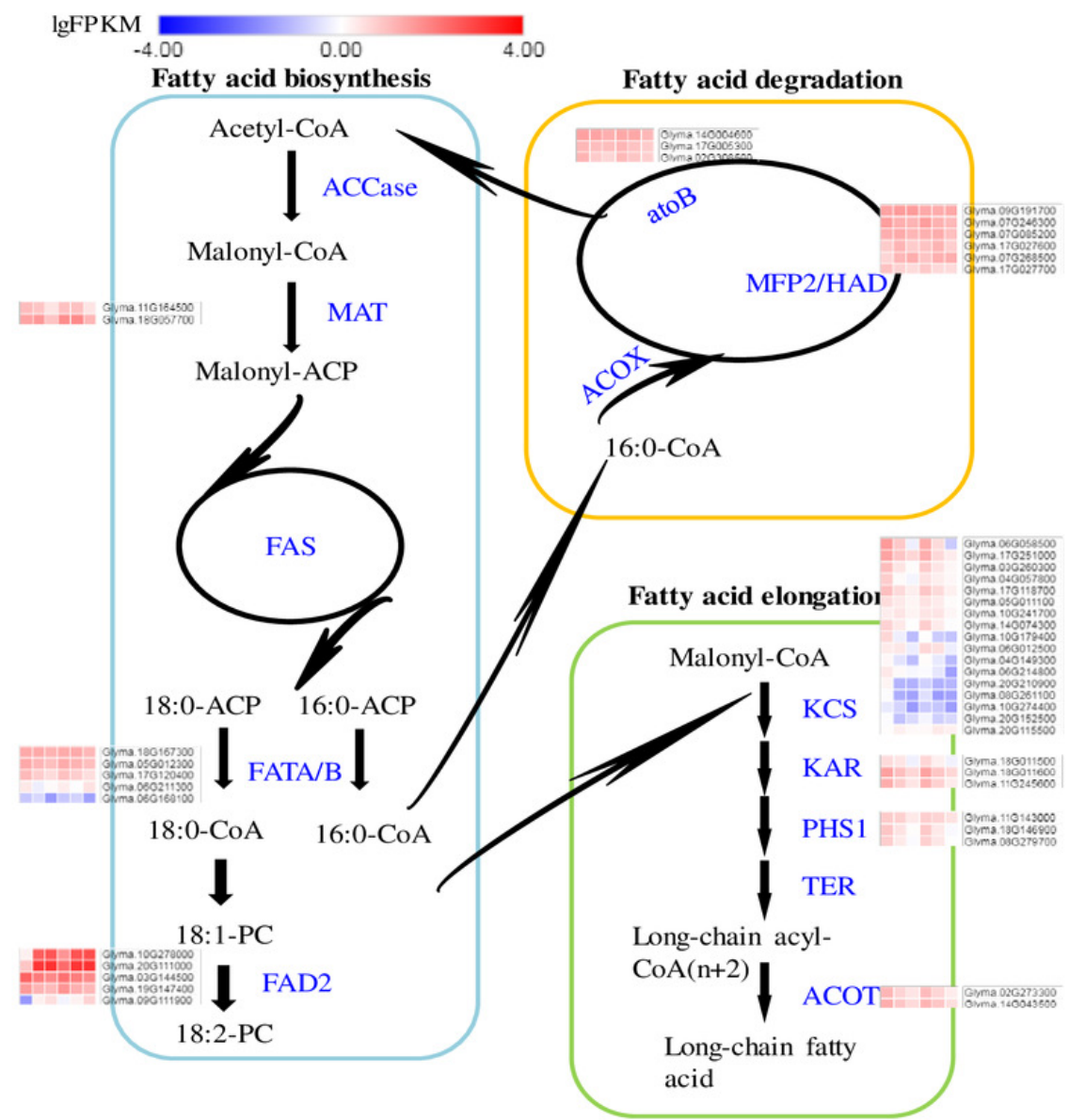




\section{Figure 7}

Figure 7. qRT-PCR analysis of DEGs with different samples from that in RNAseq.

X-axis represented the developmental stages, the black columns represented qRT-PCR results, and the red dots represented RNA-Seq results; Y-axis represented the relative level of gene expression in qRT-PCR (left) and FPKM in RNA-Seq (right). 

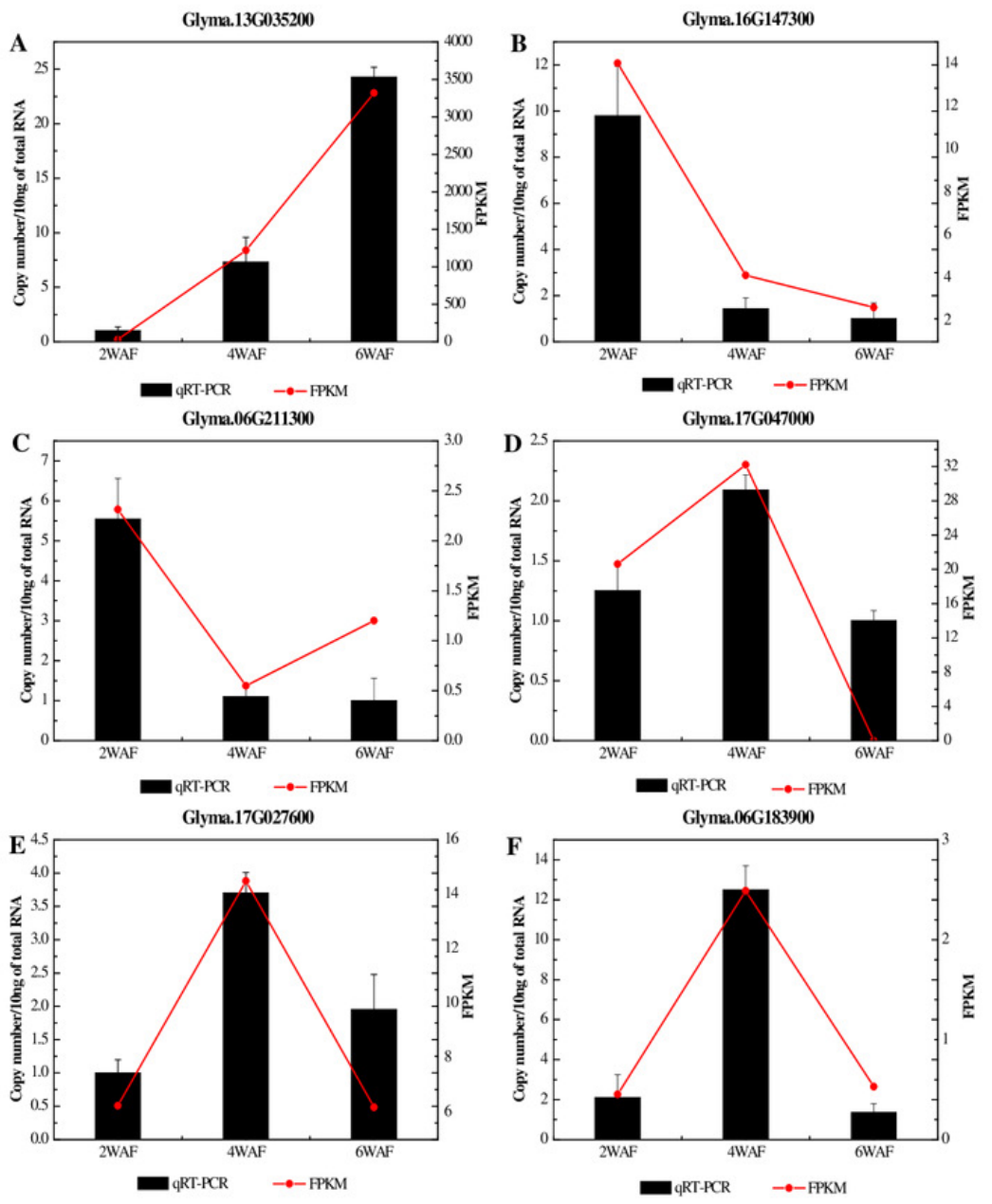


\section{Table $\mathbf{1}$ (on next page)}

Table 1. Summary of soybeans seeds transcriptome data sequenced by the Illumina platform 
1 Table 1. Summary of soybeans seeds transcriptome data sequenced by the Illumina platform

\begin{tabular}{cccccccc}
\hline Sample & A35-2WAF & A35-4WAF & A35-6WAF & A7-2WAF & A7-4WAF & A7-6WAF & Sum/Ave \\
\hline Raw reads & $45,623,515$ & $48,593,831$ & $46,469,858$ & $60,380,729$ & $49,145,343$ & $44,862,351$ & $295,075,627$ \\
Raw bases & $6,843,527,200$ & $7,289,074,700$ & $6,970,478,700$ & $9,057,109,400$ & $7,371,801,500$ & $6,729,352,700$ & $44,261,344,200$ \\
Q20(\%) & 95.97 & 96.89 & 96.77 & 96.25 & 96.13 & 96.46 & 96.41 \\
Q30(\%) & 90.78 & 92.57 & 92.32 & 91.06 & 90.98 & 91.70 & 91.57 \\
Clean reads & $45,229,794$ & $48,357,101$ & $46,210,171$ & $59,926,314$ & $48,762,965$ & $44,562,063$ & $293,048,408$ \\
Clean bases & $6,703,514,302$ & $7,186,695,710$ & $6,868,762,709$ & $8,891,051,948$ & $7,243,899,179$ & $6,621,751,911$ & $43,515,675,759$ \\
Q20(\%) & 96.42 & 97.20 & 97.13 & 96.78 & 96.59 & 96.83 & 96.83 \\
Q30(\%) & 91.40 & 92.99 & 92.81 & 91.80 & 91.62 & 92.20 & 92.14 \\
Mapped reads & 39120215 & 44375847 & 41099599 & 54665387 & 44537617 & 40763561 & $264,562,226$ \\
Proportion (\%) & 86.64 & 91.56 & 89.61 & 91.17 & 91.30 & 91.50 & 90.30 \\
\hline
\end{tabular}

2 